\title{
Sentiment Analysis of Children and Youth Literature: Is There a Pollyanna Effect?
}

\author{
Arthur M. Jacobs ${ }^{1,2 *}$, Berenike Herrmann ${ }^{3}$, Gerhard Lauer ${ }^{3}$, Jana Lüdtke ${ }^{1,2}$ and \\ Sascha Schroeder ${ }^{4}$ \\ ${ }^{1}$ Department of Experimental and Neurocognitive Psychology, Freie Universität Berlin, Berlin, Germany, ${ }^{2}$ Center \\ for Cognitive Neuroscience Berlin (CCNB), Freie Universität Berlin, Berlin, Germany, ${ }^{3}$ Digital Humanities Lab, Universität \\ Basel, Basel, Switzerland, ${ }^{4}$ Educational Psychology, University of Göttingen, Göttingen, Germany
}

OPEN ACCESS

Edited by: Ludovic Ferrand,

Centre National de la Recherche Scientifique (CNRS), France

Reviewed by: Chris Danforth,

University of Vermont, United States Eric Michael Clark,

University of Vermont, United States

*Correspondence:

Arthur M. Jacobs

ajacobs@zedat.fu-berlin.de

Specialty section:

This article was submitted to Language Sciences, a section of the journal

Frontiers in Psychology

Received: 21 June 2020

Accepted: 17 August 2020

Published: 24 September 2020

Citation:

Jacobs AM, Herrmann B, Lauer G, Lüdtke J and Schroeder S (2020) Sentiment Analysis of Children and Youth Literature: Is There a Pollyanna Effect?

Front. Psychol. 11:574746. doi: 10.3389/fpsyg.2020.574746
If the words of natural human language possess a universal positivity bias, as assumed by Boucher and Osgood's (1969) famous Pollyanna hypothesis and computationally confirmed for large text corpora in several languages (Dodds et al., 2015), then children and youth literature $(C Y L)$ should also show a Pollyanna effect. Here we tested this prediction applying an unsupervised vector space model-based sentiment analysis tool called SentiArt (Jacobs, 2019) to two CYL corpora, one in English (372 books) and one in German (500 books). Pitching our analysis at the sentence level, and assessing semantic as well as lexico-grammatical information, both corpora show the Pollyanna effect and thus add further evidence to the universality hypothesis. The results of our multivariate sentiment analyses provide interesting testable predictions for future scientific studies of literature.

Keywords: Pollyanna effect, positivity superiority effect, sentiment analysis, SentiArt, neurocognitive poetics, affective-aesthetic potential, digital humanities, children and youth literature

"The 7-year-old child is perfectly familiar with both PRETTY and UGLY but he uses the former much more frequently than the latter." Boucher and Osgood (1969, p. 7).

\section{INTRODUCTION}

In 1969 Boucher and Osgood presented influential evidence for the idea that "humans tend to look on (and talk about) the bright side of life" and coined this phenomenon the "Pollyanna hypothesis," i.e., a universal human tendency to use evaluatively positive words more frequently, diversely and facilely than evaluatively negative words ${ }^{1}$. About 50 years and many technological advances later - especially in natural language processing (NLP), computational linguistics and machine learning methods - Dodds et al. (2015, p. 6) presented extensive cross-cultural data based on largescale macroanalytic, univariate sentiment analyses of multi-lingual text corpora that support the hypothesis. They concluded their study with: "Overall, our major scientific finding is that when experienced in isolation and weighted properly according to use, words, which are the atoms of human language, present an emotional spectrum with a universal, self-similar positive bias. We emphasize that this apparent linguistic encoding of our social nature is a system-level property, and

\footnotetext{
1 "Pollyanna Grows Up," a 1915 novel by the American author Eleanor H. Porter, is considered a classic of children's literature and POLLYANNA has become a byword for someone who - like the title character - has an unswervingly optimistic attitude with a clear bias toward the positive.
} 
in no way asserts all natural texts will skew positive... or diminishes the salience of negative states (Forgas, 2013). Going forward, our word happiness assessments should be periodically repeated and carried out for new languages, tested on different demographics, and expanded to phrases both for the improvement of hedonometric instruments and to chart the dynamics of our collective social self." In a similar vein, Greene (2017, p.12) found hints to a positivity bias in his summary of text analyses of the "corpus of the canon of western literature" concluding "that even though canonical literature from Homer to Hemmingway addresses death, war, heartache and tragedy, the overall cultural preoccupations of the western canon over history have been largely positive."

Such a textual positivity bias has measurable consequences for text processing and reading behavior known as the positivity superiority effect, i.e., the observation that in many word recognition tasks positive words yield faster response times than neutral or negative ones (Lüdtke and Jacobs, 2015; for review, see Jacobs et al., 2015). This effect, which has also been observed in 6-12 year old children (Sylvester et al., 2016), is usually explained with the informational density hypothesis (Ashby and Isen, 1999; Kuchinke et al., 2005; Unkelbach et al., 2010). It claims that positive information is generally processed faster on grounds of subjective exposure frequency, i.e., the experienced frequency with which positive information is internally activated in memory. Taking subjective exposure frequency as a proxy for higher informational density of lexical representations of positive words thus would cause them to be processed faster because they are better elaborated and interconnected in memory. Indeed, there is neurocomputational evidence for the idea that positive words provide more and denser semantic longterm associations than neutral or negative ones (Hofmann and Jacobs, 2014) which has been related to the hippocampus being more generally involved in the processing of positive affect (Hofmann and Kuchinke, 2015).

\section{THE PRESENT STUDY}

Both Dodds et al.'s (2015) and Greene's (2017) studies used texts for adults. Inspired by the above citation from Boucher and Osgood (1969) and previous behavioral studies from our group also supporting the Pollyanna hypothesis (Sylvester et al., 2016), here we were interested in finding out to what extent international texts of children and youth literature (CYL) also show the Pollyanna effect, including the book who's protagonist coined the effect (Porter, 1915). For this purpose, we submitted Porter's book, as well as 372 English and 500 German books representing CYL, to a computational sentiment analysis using the empirically well validated SentiArt tool which is based on (semantic) vector space models/VSM (Jacobs, 2019; Jacobs and Kinder, 2019).

Given that so far the SentiArt tool was only used for the analysis of rating and reading data of adult persons, we first cross-validated it with valence rating data from the kidBawl study (Sylvester et al., 2016). We then report the results of the computational sentiment for Porter's (1915) book "Pollyanna
Grows Up" before applying SentiArt to two large CYL corpora in both English and German.

\section{CORPORA}

The textual data used in this study come from two published corpora, the Gutenberg Literary English Corpus (GLEC; Jacobs, 2018b) and the (German) childLex corpus (Schroeder et al., 2015). Since both have been extensively described in the aforementioned papers, here we just give a brief summary of GLEC-CYL and childLex. The GLEC-CYL corpus is a subset of GLEC, containing 372 books from 25 different authors such as Beatrix Potter, Lyman Frank Baum or RM Ballantyne. For copyright reasons this corpus contains only books published before 1952. In contrast, the 500 books in the German childLex corpus mainly contain post-war and contemporary exemplars such as the seven books from the Harry Potter series (e.g., Rowling, 1997) and include a nice mix of texts by a large variety of well-known and less well-known German and translated international writers $(N=248)$ like Alexandre Dumas, Kirsten Boie, Erich Kästner, Ottfried Preussler, Enid Blyton, or Antoine de Saint-Exupeiry. Table 1 shows 10 example books from each corpus. The texts in both corpora were preprocessed using standard python NLP tools, i.e., words were POS-tagged using treetagger ${ }^{2}$ and only content words (nouns, verbs, adjectives, and adverbs) were kept for the sentiment analyses using SentiArt.

\section{METHODS}

\section{Sentiment Analysis}

Dodds et al. (2015) confirmed the Pollyanna hypothesis in large text corpora (e.g., google books, movie subtitles, and twitter) in different languages with a special word-list-based sentiment analysis ("hedonometer") which selects the most frequent 510,000 words only. When analyzing single books, they also used a special method sliding a 10,000-word window through each book and computing the average univariate "happiness score." Using a "lens" for their hedonometer to obtain a strong signal, they excluded all words for which $3<$ happiness score $<7$ (i.e., they kept words residing in the tails of each distribution going from 1 to 9 ).

Our approach using SentiArt is different. Instead of using word lists based on human valence ratings - a procedure which presents a number of both methodological and epistemological problems when trying to cross-validate the predictions of a sentiment analysis tool with other human rating data (Hollis et al., 2017; Hofmann et al., 2018) - SentiArt is based on VSMs. Using VSMs offers several advantages discussed in previous articles (Jacobs, 2019; Jacobs and Kinder, 2019), such as avoiding these problems and being applicable to any language for which VSMs are publically available (e.g., the $>120$ VSMs of fasttext ${ }^{3}$ ).

Unlike most sentiment analysis tools, SentiArt computes a multivariate sentiment analysis offering a dozen affective

\footnotetext{
${ }^{2}$ https://www.cis.uni-muenchen.de/\$ $\backslash$ sim $\$$ schmid/tools/TreeTagger/
}

${ }^{3}$ https://fasttext.cc/docs/en/pretrained-vectors.html 
TABLE 1 | 10 example texts from both corpora.

\section{GLEC-CYL}

Andrew Lang. Tales of Troy and Greece

Baronness Orczy. The Scarlet Pimpernel

Beatrix Potter. The Tale Of Peter Rabbit

Edward Stratemeyer. The Rover Boys in the Land of Luck

Jacob Abbott. Cleopatra

James Matthew Barrie. Peter Pan

Louisa May Alcott. Rose in Bloom

Lyman Frank Baum. The Wonderful Wizard of Oz

R M Ballantyne. Away in the Wilderness

Thornton Waldo Burgess. Mrs. Peter Rabbit

\section{ChildLex}

Kirsten Boie. King Kong das Schulschwein

Michael Ende. Momo

Cornelia Funke. Tintenherz

Martin Klein. Der Geist aus dem Würstchenglas

Max Kruse. Urmel aus dem Eis

Paul Maar. Sams in Gefahr

Joanne K. Rowling. Harry Potter und der Stein der Weisen

Ottfried Preussler. Die kleine Hexe

Antoine de Saint-Exupeiry. Der Kleine Prinz

Nils Werner. Teddy Brumm

TABLE 2 | Results of sentiment analyses for GLEC-CYL and childLex.

\begin{tabular}{|c|c|c|c|c|}
\hline Feature & Meaning & Computation & GLEC-CYL & ChildLex \\
\hline Sentences & Average number of sentences / book & NLTK sentence tokenizer & $2,402 \pm 68$ & $1,116 \pm 68.1$ \\
\hline Words/sentence & Average number of words / sentence & NLTK word tokenizer & $8.0 \pm 0.12$ & $5.9 \pm 1.4$ \\
\hline Tokens & Average number of tokens / book & NLTK word tokenizer & $18,589 \pm 552$ & $7,351 \pm 510$ \\
\hline Types & Average number of types / book & NLTK word tokenizer & $4,217 \pm 105$ & $2,341 \pm 106$ \\
\hline Word length & Average number of letters / word & NLTK word tokenizer & $6.1 \pm 0.02$ & $6.5 \pm 0.01$ \\
\hline Type token ratio & $\begin{array}{l}\text { Quotient of average number of types and } \\
\text { tokens / book }\end{array}$ & NLTK word tokenizer & $0.26 \pm 0.005$ & $0.4 \pm 3.005$ \\
\hline AAP & Affective-Aesthetic Potential (see Footnote 2) & SentiArt, VSM-based using 120 labels & $0.29 \pm 0.007$ & $0.06 \pm 0.004$ \\
\hline AAP noun & AAP for nouns only & As AAP & $0.21 \pm 0.0009$ & $0.22 \pm 0.007$ \\
\hline AAP verb & AAP for verbs only & As AAP & $0.26 \pm 0.0005$ & $-.04 \pm 0.004$ \\
\hline AAP adjective & AAP for adjectives only & As AAP & $0.71 \pm 0.01$ & $0.17 \pm 0.006$ \\
\hline AAP adverb & AAP for adverbs only & As AAP & $0.50 \pm 0.005$ & $0.05 \pm 0.003$ \\
\hline Anger & $\begin{array}{l}\text { Average semantic relatedness between content } \\
\text { words and label 'anger' }\end{array}$ & SentiArt, VSM-based using 1 label only & $0.25 \pm 0.009$ & $0.26 \pm 0.0004$ \\
\hline Disgust & As anger & As anger & $0.19 \pm 0.007$ & $0.15 \pm 0.005$ \\
\hline Fear & As anger & As anger & $0.59 \pm 0.008$ & $0.54 \pm 0.005$ \\
\hline Happiness & As anger & As anger & $0.45 \pm 0.009$ & $0.53 \pm 0.005$ \\
\hline Sadness & As anger & As anger & $0.30 \pm 0.008$ & $0.24 \pm 0.005$ \\
\hline Surprise & As anger & As anger & $0.87 \pm 0.009$ & $0.58 \pm 0.005$ \\
\hline PNR & Ratio of positive/negative words per sentence & As AAP & $2.3 \pm 0.03$ & $1.5 \pm 0.02$ \\
\hline
\end{tabular}

Values are standardized means (z values) \pm 1 SE.

semantic features (see Table 2) and is empirically validated with diverse experimental data. For example, its affective-

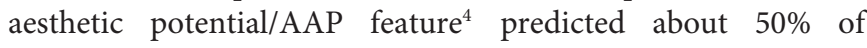
variance in human valence ratings for $>2,500$ single words and about $45 \%$ of variance in "liking" ratings for entire sections from a mystery story (Jacobs and Kinder, 2019). SentiArt also achieved $100 \%$ accuracy in predicting the sentiment category of 120 excerpts from the Harry Potter books (Jacobs, 2019) outperforming two standard sentiment

\footnotetext{
${ }^{4} \mathrm{AAP}$ refers to the average semantic relatedness between each word in a text and $m$ positive labels (lpos_1-60 = affection, amuse,..., unity) minus the average relatedness between each word and $n$ negative labels (lneg_1-60 = abominable,... , ugly). The labels were published in earlier papers (Jacobs, 2017, 2018b). For English, the semantic relatedness between a test word and each of the 120 labels is computed using the GLEC vector space model/VSM (Jacobs, 2018b) which was created applying the fasttext algorithm (https://fasttext.cc/) to the ensemble of texts in GLEC. The final model contained a 500d skipgram vector for each word from GLEC ordered by frequency of occurrence. The cosine of two word vectors gives the semantic relatedness value. For German, the VSM was the 300d skipgram SDEWAC/subtlex model (see Jacobs and Kinder, 2019; Table 1).
}

analysis tools, VADER (Hutto and Gilbert, 2014) and HU-LIU (Hu and Liu, 2004).

Since previous studies showed that the AAP feature fared better at predicting valence or liking ratings than the valence feature, it also computes, AAP will be used in the following analyses. Higher AAP values theoretically indicate a word's or text's higher potential for evoking positive affective responses, including aesthetic feelings of liking and beauty. Thus, the AAP was the most important feature in a recent empirical study showing that human beauty ratings for single words can perfectly be classified via machine learning on the basis of a total of eight quantitative word features (Jacobs, 2017). In the following analyses, the AAP feature is complemented by six discrete emotion features (anger, disgust, fear, happiness, sadness, and surprise) based on classic emotion theories (for review, see Westbury et al., 2015). SentiArt computes these six features (similarly to AAP) via a VSM-based procedure using single labels (e.g., the word "happiness") instead of sets comprising 60 items. Thus, the "happiness" score of a sentence, for example, 
corresponds to the average semantic relatedness between each content word and the word "happiness," as computed via the cosine between the corresponding word vectors.

From a psychological, reader response, or neurocognitive poetics perspective, two questions are important. First, on which unit(s) of text a normal reader bases her affective-aesthetic appreciation of an entire book or book chapter: words, sentences, paragraphs, or perhaps pages? The answer to this first question is unknown and a constant challenge to researchers in the emerging fields of neurocognitive poetics (Jacobs, 2015a; Willems and Jacobs, 2016; Nicklas and Jacobs, 2017). For practical reasons, when running empirical studies on whole books, the sentence has appeared to be the smallest viable unit. For example ${ }^{5}$, Jockers (2017) had four readers rate each sentence's valence of several contemporary novels and found correlations ranging from.52 to. 83 between the predictions of his sentiment analysis tool (i.e., the word list based Syuzhet) and the readers' ratings, depending on the novel. Other researchers had readers rate the valence of "story sections" (corresponding roughly to paragraphs; Lehne et al., 2015) or each sentence of book chapters (Jacobs et al., 2020). We are not aware of studies of entire books using readers' valence rating on word level and thus follow Jockers in selecting the sentence as the basic unit. Note, however, for Bestgen's (1994) seminal study, readers rated about $40 \%$ of the unique content words (types) of certain shorter texts (e.g., Hans Christian Andersen's "The little match girl”), albeit not in the story context.

A sentence-based procedure of course raises the second question whether the average word valence is the optimal estimate for a sentence's valence. Bestgen's (1994, Table 2) correlational data - the most informative for answering this question, as far as we can tell - suggest that lexical (word) valence predicted between 30 and $60 \%$ of the variance in (average) sentence valence, while (average) sentence valence predicted between 60 and $70 \%$ of the variance in text valence, depending on the text.

Given these results, it seemed most promising to compute the mean valence (or, in our case, AAP) averaged across all content words (nouns, verbs, adjectives, and adverbs) of a sentence. In addition, we also computed mean AAP sentence values based on distinct word types only, e.g., nouns or adjectives. This is because it is so far unknown to what extent different word types (lexico-grammatical information) may contribute to the emotional evaluation of a sentence. The data by Lüdtke and Jacobs (2015) suggested non-linear interactive effects between the valence of nouns and adjectives on emotional evaluations of simple short declarative sentences (e.g., "The grandpa is lonely"). They showed that negative adjectives dominated supralexical evaluation, which can be interpreted as a sort of negativity bias. Since so far, no empirical follow-up studies have investigated more complex sentences or the influence of other word types (e.g., verbs and adverbs), we do not know whether Lüdtke and Jacobs' findings may generalize to complex sentences. This is important because the bulk of sentences contained in our literature corpora are complex ones. In addition to the overall mean sentence AAP, and mean noun-, verb-, adjective- and

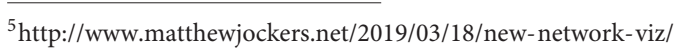

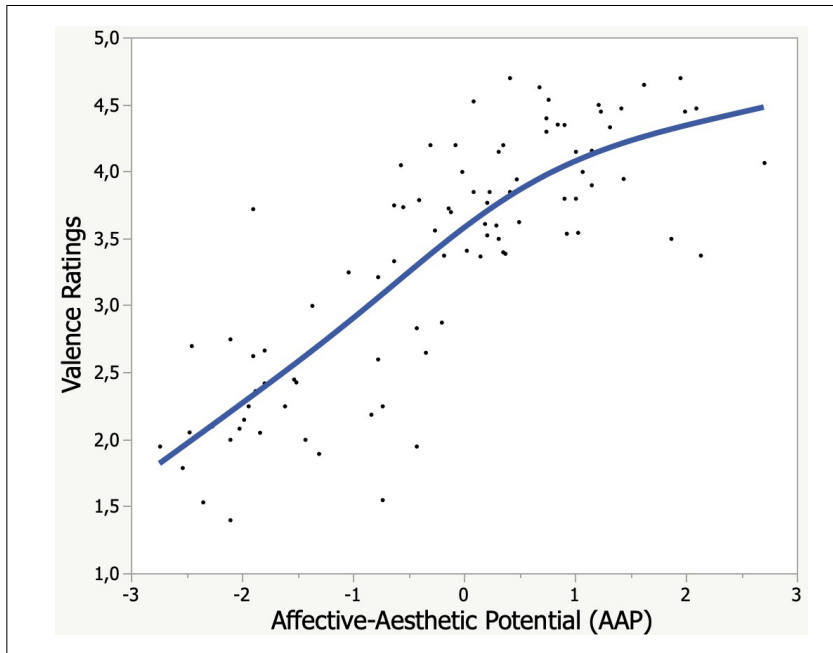

FIGURE 1 | Rated word valence as a function of computational affective-aesthetic potential (AAP).

adverb-based AAP, we also computed the ratio between the frequency of positive (AAP value $>0$ ) and negative words (AAP value $<0$ ) per sentence (the PNR). The PNR allows answering the question whether positive words were used more often in a book than negative words, as hypothesized by Boucher and Osgood (1969). Finally, we computed the semantic relatedness of each content word in a sentence with each of the six basic emotions (i.e., anger, disgust, fear, happiness, sadness, and surprise) and the corresponding mean per sentence. Thus, for each sentence of every book 12 affective semantic features went into the present sentiment analyses (see Table 2).

\section{RESULTS AND DISCUSSION}

\section{Study 1: Cross-Validation of SentiArt With Human Rating Data From the kidBAWL}

The predictive validity of the AAP feature computed by SentiArt was tested with the kidBAWL valence rating data from Sylvester et al.'s (2016) Experiment 1, where six to 12 years old children read a subset of 90 words from the kidBAWL and judged the word's valence on a 5-point scale (very unpleasant - unpleasant neither unpleasant nor pleasant - pleasant - very pleasant). The results of the cross validation shown in Figure 1 support those of the aforementioned studies, establishing a good empirical predictive validity of SentiArt for human rating data, yielding an $R_{a d j}^{2}=0.68$ (logistic fit; linear fit: $R_{a d j}^{2}=0.65$ ). Together with the findings of previous studies (Westbury et al., 2015; Hollis et al., 2017; Hofmann et al., 2018; Jacobs, 2019; Jacobs and Kinder, 2019) this offers even more evidence supporting the validity of VSM-based sentiment analysis tools which, in contrast to word list based tools, cannot be criticized for the aforementioned epistemological or psychometric problems. Having shown the validity of SentiArt with rating data from children of age 7 to 12 , we now proceed with the computational text analyses. 


\section{Study 2: Sentiment Analysis of Porter's (1915) "Pollyanna Grows Up"}

Before we proceed to test whether CYL at large indeed exhibits the "Pollyanna" effect (as suggested by preliminary research), we think it is quite natural to ask whether the effect is exhibited by the very book whose protagonist became emblematic of it. The following computational data based on SentiArt's AAP feature suggest a "yes" answer. The wordcloud in Figure 2A gives a first idea why the book is - on average - more positive than negative. The cloud summarizes data of the 1,000 most positive and 1,000 most negative words in the book and positive words like NEW or LOVELY (the words with the highest frequency of occurrence among the 2,000; $N=53$ and 43, respectively) clearly dominate negative ones like AFRAID or CRY ( $N=25$ and 22, respectively). More detailed evidence suggesting that the Pollyanna hypothesis is borne out is shown in Figures 2B,C. The "emotional time series" in Figure 2B shows a profile which resembles the typical "man in hole" emotional arc profile (Vonnegut, 1981; Reagan et al., 2016), except for the final fall. The fact that most of the area of the smoothed curve lies above the zero line indicates an overall positive AAP. Figure 2C corroborates the positivity bias with histogram data which have a mean AAP value of 0.4 for 4,125 sentences $^{6}$. Although showing the effect in the very book

${ }^{6} \mathrm{~A} z$-test established that the mean value of 0.4 is significantly greater than 0 $(z=31.7, p<0.0001)$. that coined its name may not come as a big surprise, this finding yields another, if slightly informal, type of validation of the said effect and thus is a good start for the upcoming analyses of the 372 GLEC-CYL and 500 childLex books.

\section{Study 3: Sentiment Analysis of GLEC-CYL and ChildLex}

Here we computed the 12 affective semantic features outlined in Table 2 for our two corpora using SentiArt together with other text features indicating style such as type token ratio (Jacobs, 2018a). The mean hit-rate or coverage (i.e., the overlap between the VSM's vocabulary and all content words in all books) was $98 \%$ for GLEC-CYL and $88 \%$ for childLex.

The global statistics for the books from two CYL corpora given in Table 2 can be summarized as follows. On average, the English books are longer than the German ones, having more sentences and more words per sentence. Note, however, that these comparisons are only suggestive and cannot be generalized without further investigations given the enormous differences in publication period or number of different authors between the two corpora.

Regarding the sentiment analysis, GLEC-CYL books generally adhere to the Pollyanna principle exhibiting a positivity bias for all AAP values and a ratio of positive/negative words per sentence (PNR) of 2.3 per sentence, i.e., on average there are clearly more positive words than negative ones in a sentence. At the
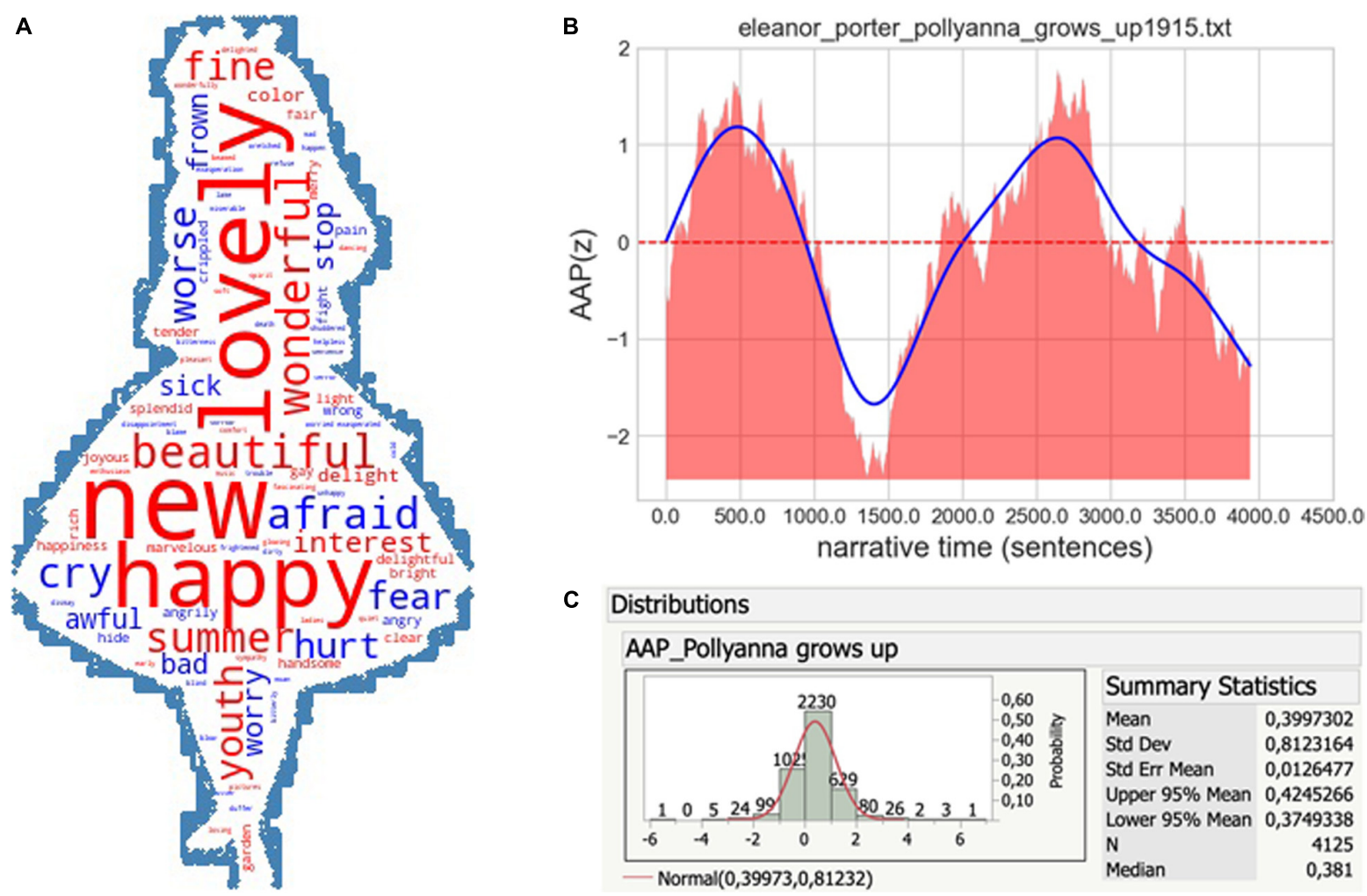

C Distributions

AAP_Pollyanna grows up

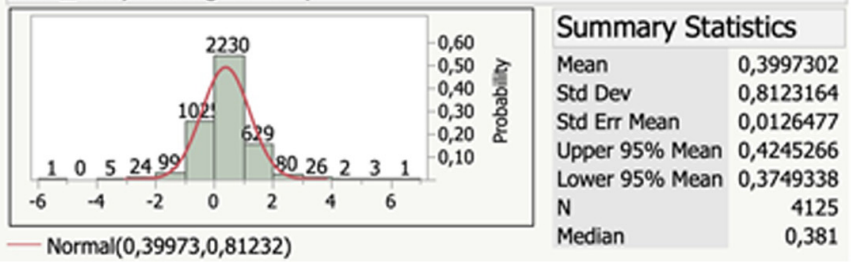

FIGURE 2 | (A-C) Wordcloud, emotional (narrative) time series and affective-aesthetic potential (AAP) distribution for "Pollyanna grows up." The blue curve in (B) represents the smoothed average, the dotted red line the zero border. 
level of semantic relations with discrete emotion words, GLECCYL books are dominated by surprise, fear, and happiness, while sadness, anger and disgust play minor roles. With regard to the abovementioned issue of a possible negativity bias due to nounadjective interactions (Lüdtke and Jacobs, 2015) the GLEC-CYL data rather indicate, at least on average, a positivity bias, since both "AAP noun" and "AAP adjective" features are positive.

Just as the English corpus, the books from childLex also generally adhere to the Pollyanna principle. Note, though, that the affective semantic feature values from the two corpora are not directly comparable, since they stem from different VSMs. Similarly to GLEC-CYL, childLex exhibits a general positivity bias $^{7}$ - except for AAP verb - and with a PNR of 1.5 per sentence a clear signal of more positive words at the sentence level. Regarding discrete emotion words, we find the same pattern as for GLEC-CYL with surprise, fear, and happiness dominating in childLex books, while sadness, anger and disgust play minor roles. Again, there is no general indication of a possible negativity bias due to noun-adjective interactions, both being congruently positive on average.

The distributional data in Figure 3 show that a single book in GLEC-CYL had an overall negative AAP (Beatrix Potter's “The Story of Miss Moppet"), while in childLex 142 books exhibit an overall negativity bias $(\sim 30 \%)$. Again, the results for the very homogeneous GLEC-CYL in which $341 / 372$ books stem from only nine different authors cannot directly be compared, though,

${ }^{7} \mathrm{~A} z$-test established that the mean value of 0.06 is significantly greater than 0 $(z=14.0, p<0.0001)$

\section{A Distributions}

\section{AAP}

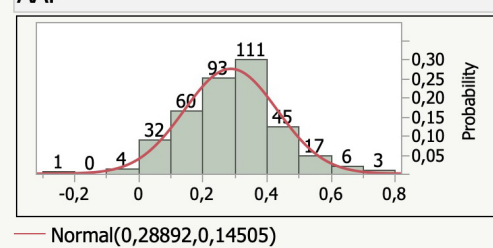

Happiness

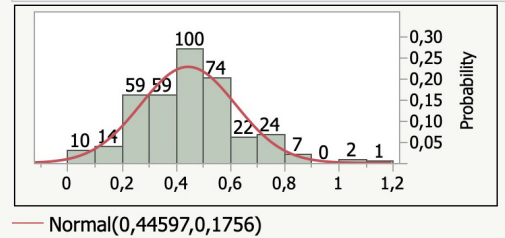

PNR

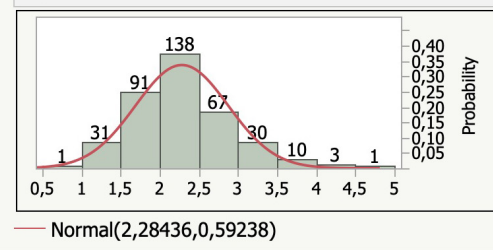

Summary Statistics

Mean $\quad 0,2889247$ Std Dev $\quad 0,1450545$ Std Err Mean $\quad 0,0075207$ Upper $95 \%$ Mean 0,3037133 Lower 95\% Mean 0,2741361

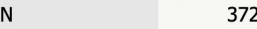

$\begin{array}{lr}\text { Median } & 372 \\ & 0,297\end{array}$

\section{Summary Statistics}

Mean $\quad 0,4459731$

Std Dev $\quad 0,1756036$ Std Err Mean $\quad 0,0091046$ Upper $95 \%$ Mean 0,4638763 Lower $95 \%$ Mean $\quad 0,42807$ $\begin{array}{lr}\mathrm{N} & 372\end{array}$ Median $\quad 0,452$

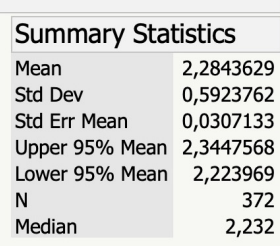

to those of childLex. Thus, we can only propose two heuristic hypotheses: there might be (a) a more pronounced positivity bias in English CYL when compared to German CYL; (b) a trend toward a less pronounced positivity bias from $19^{\text {th }}$ century to contemporary CYL. Both hypotheses (and possible interactions) need to undergo further testing in future studies.

What is relatively safe to formulate as a testable hypothesis is that in both corpora readers have a higher probability of positive feelings associated with surprise than for negative feelings associated with disgust. Readers of books from both corpora also face a theoretically high probability of experiencing thoughts or feelings associated with fear. According to our sentiment analyses, this probability would be highest for the following three books from the GLEC-CYL corpus: James Matthew Barrie's “Tommy and Grizel," Louisa May Alcott's "Pauline's Passion and Punishment," and Thornton Waldo Burgess' "Lightfoot the Deer" which all showed 'fear' scores of $>1$. In childLex, it would be: Knister's (Ludger Jochmann), "Hexe Lilli und der verflixte Gespensterzauber, as well as Sabine Neuffer's "Lukas und Felix werden Freunde" and Kirsten Boie's, "King Kong das Krimischwein" (fear score $>0.9$ ). When discussing these findings, incorporating contextual information is crucial. It is thus an open empirical question whether - within the appropriate reading context (paragraph and chapter), and not in isolationa single sentence from Barrie's book like "Young man, I fear you are doomed," for which the relatively highest fear score was computed, really induces higher, "fear" ratings than other sentences, or whether, it is rather the book as a whole that has a higher probability of being associated with fear feelings

\section{B Distributions}

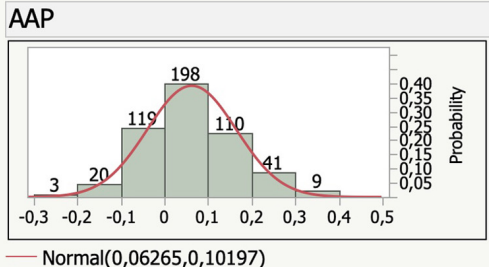

Summary Statistics Mean $\quad 0,062648$ Std Dev $\quad 0,1019661$ Std Err Mean $\quad 0,0045601$ Upper $95 \%$ Mean 0,0716073 Lower $95 \%$ Mean 0,0536887 N Median 0,053 Happiness

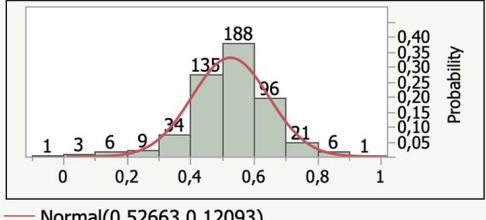
\begin{tabular}{l|r}
\hline \multicolumn{2}{l}{ Summary Statistics } \\
\hline Mean & 0,526628 \\
Std Dev & 0,1209333 \\
Std Err Mean & 0,0054083 \\
Upper 95\% Mean & 0,5372538 \\
Lower 95\% Mean & 0,5160022 \\
N & 500 \\
Median & 0,535
\end{tabular} PNR

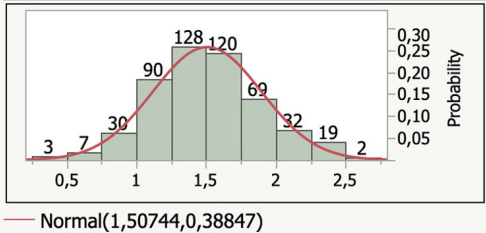

FIGURE 3 | (A,B) Affective-aesthetic potential (AAP), happiness score and ratio of positive/negative words per sentence (PNR) distributions for GLEC-CYL (left) and childLex (right). 
(when compared to other books). In addition, the feeling that a single sentence may induce will depend on a mix of different scores, e.g., to what extent a word having a high fear score is in the company of words having high happiness or sadness scores, and as well on the mean AAP value. Moreover, also the question whether mean AAP will be the best approximation of sentence AAP or whether some kind of word type by valence interaction has to be taken into account (cf. Lüdtke and Jacobs, 2015), have to be answered by future studies. We believe that predictive modeling studies using advanced computational text analysis tools like SentiArt (e.g., Jacobs, 2017; Xue et al., 2019, 2020) are not the only but surely a very promising way of finding out which of these possibilities come near to reality, which, of course, also involves effects of the preceding and following context, and of reader personality factors such as mood (Lüdtke et al., 2014; Jacobs et al., 2016b).

Thus, being based on computational models, the present sentiment analyses, like others, cannot provide a necessity, but only a sufficiency analysis, i.e., they are a tool for quantitatively predicting how things could be, if certain conditions hold. Whether this corresponds to reality must be determined via adequate empirical testing which then can inform the improvement of computational sentiment analysis tools, e.g., by indicating that the present six discrete emotion scores are not sufficient for good reader response predictions and should be augmented (or replaced) by other scores. It should be noted, though, that apart from the aforementioned behavioral studies cross-validating predictions from SentiArt there is also neuroimaging evidence indicating that text passages from the Harry Potter books which have a high theoretical "fear" potential can indeed activate brain regions associated with fear induction (Hsu et al., 2015).

\section{CONCLUSION, LIMITATIONS, AND OUTLOOK}

Despite differences in the computational methods, the present sentiment analysis results support the findings reported by Dodds et al. (2015), showing that also international classical and contemporary CYL generally exhibits the Pollyanna principle as hypothesized by Boucher and Osgood (1969). Both an English corpus from the $19^{\text {th }}$ century with only 25 different authors and a contemporary German corpus with $>200$ different authors clearly show a positivity bias, not only in a single text feature (as, e.g., in Dodds et al.'s univariate sentiment analysis), but in a variety of features such as AAP, AAP noun, happiness, or PNR. Together with those of previous cross-validation studies (e.g., Jacobs and Kinder, 2019) the results of the present Study 1 are promising, accounting for almost $70 \%$ of valence ratings. However, at the same time, they still leave $\sim 30 \%$ of variance unaccounted for. This could be due to the unknown experiential/embodied part of affective semantics that cannot be captured by distributional semantics models like the VSMs used here (Jacobs et al., 2016a), or, of course to other possible limitations of SentiArt, e.g., regarding the choice of labels. When applying lexical sentiment values to larger units of text (i.e., sentences and chapters) the amount of unaccounted variance can, but must not necessarily, increase nonlinearly, as can be inferred from the correlations obtained by the aforementioned studies by Bestgen (1994) and Jockers (yielding $R^{2}$ values for sentences between 0.27 and 0.7 , depending on the text). There is definitely a long way to go before a fuller understanding of the processes underlying readers' affective-aesthetic text evaluation is achieved. Combined efforts complementing quantitative ("distant") digital humanities with close reading studies are necessary, as are greater efforts in developing adequate training corpora, VSMs and empirical designs combining direct offline with indirect online methods of scientific studies of literature (Dixon and Bortolussi, 2015; Jacobs, 2015b; Kuiken, 2015). Reporting on a variety of distinct measures for gauging sentiment and emotion for "positivity" in young readers' books, our study has shown that a widely discussed phenomenon such as the Pollyanna effect can - and in fact should - undergo further nuanced theoretical and computational modeling. In addition to the more "standard" univariate measures, our unsupervised multivariate approach theoretically allows for a more nuanced modeling of aesthetic emotions in literature reception. As these represent readers' interaction with the "poetic form" (Jakobson, 1960), their incorporation offers a more refined account of the affective ecology of literary reading, and thus a deeper grasp of "positivity" encoded in our cultural heritage.

\section{DATA AVAILABILITY STATEMENT}

The raw data supporting the conclusions of this article will be made available by the authors, without undue reservation.

\section{AUTHOR CONTRIBUTIONS}

AJ designed the study, analyzed the data with advice from all authors, and drafted the manuscript. All authors contributed to the final version of the manuscript.

\section{FUNDING}

This research was supported by grant JA 823/12-1 (Advanced sentiment analysis for understanding affective-aesthetic responses to literary texts: A computational and experimental psychology approach to children's literature) of the SPP 2207 'Computational Literary Studies' of the German Research Foundation (DFG). Open Access Funding was provided by the Freie Universität Berlin. 


\section{REFERENCES}

Ashby, F. G., and Isen, A. M. (1999). A neuropsychological theory of positive affect and its influence on cognition. Psychol. Rev. 106:529. doi: 10.1037/0033-295x. 106.3.529

Bestgen, Y. (1994). Can emotional valence in stories be determined from words? Cogn. Emot. 8, 21-36. doi: 10.1080/02699939408408926

Boucher, J., and Osgood, C. E. (1969). The pollyanna hypothesis. J. Verb. Learn. Verb. Behav. 8: 1-8. doi: 10.1016/S0022-5371(69)80002-2

Dixon, P., and Bortolussi, M. (2015). Measuring literary experience: comment on Jacobs. Sci. Study Lit. 5, 178-182. doi: 10.1075/ssol.5.2.03dix

Dodds, P. S., Clark, E. M., Desu, S., Frank, M. R., Reagan, A. J., Williams, J. R., et al. (2015). Human language reveals a universal positivity bias. Proc. Natl. Acad. Sci. U.S.A. 112: 2389-2394.

Forgas, J. P. (2013). Don't worry, be sad! On the cognitive, motivational, and inter-personal benefits of negative mood. Curr. Dir. Psychol. Sci. 22, 225-232.

Greene, C. (2017). Introducing the corpus of the Canon of Western Literature: a corpus for culturomics and stylistics. Lang. Lit. 26, 282-299. doi: 10.1177/ 0963947017718996

Hofmann, M. J., and Jacobs, A. M. (2014). Interactive activation and competition models and semantic context: from behavioral to brain data. Neurosci. Biobehav. Rev. 46, 85-104. doi: 10.1016/j.neubiorev.2014.06.011

Hofmann, M. J., and Kuchinke, L. (2015). "Anything is good that stimulates thought" in the hippocampus Comment on "The quartet theory of human emotions: an integrative and neurofunctional model" by S. Koelsch et al. Phys. Life Rev. 13, 58-60. doi: 10.1016/j.plrev.2015.04.007

Hofmann, M. J., Biemann, C., Westbury, C., Murusidze, M., Conrad, M., and Jacobs, A. M. (2018). Simple co-occurrence statistics reproducibly predict association ratings. Cogn. Sci. 42, 1-26. doi: 10.1111/cogs.12662

Hollis, G., Westbury, C., and Lefsrud, L. (2017). Extrapolating human judgments from skip- gram vector representations of word meaning. Q. J. Exp. Psychol. 70, 1-45. doi: 10.1080/17470218.2016.1195417

Hsu, C. T., Jacobs, A. M., Citron, F., and Conrad, M. (2015). The emotion potential of words and passages in reading Harry Potter - An fMRI study. Brain Lang. 142, 96-114. doi: 10.1016/j.bandl.2015.01.011

$\mathrm{Hu}, \mathrm{M}$. , and Liu, B. (2004). "Mining and summarizing customer reviews", in Proceedings of the Tenth ACM SIGKDD International Conference on Knowledge Discovery and Data Mining, eds W. Kim and R. Kohavi (Washington, DC: ACM Press), 168-177. doi: 10.1145/1014052.1014073

Hutto, C. J., and Gilbert, E. E. (2014). "VADER: a parsimonious rule-based model for sentiment analysis of social media rext," in Proceedings of the Eighth International Conference on Weblogs and Social Media (ICWSM-14). Ann Arbor, MI

Jacobs, A. M. (2015a). Neurocognitive poetics: methods and models for investigating the neuronal and cognitive-affective bases of literature reception. Front. Hum. Neurosci. 9:186. doi: 10.3389/fnhum.2015.00186

Jacobs, A. M (2015b). The scientific study of literary experience: sampling the state of the art. Sci. Study Lit. 5, 139-170. doi: 10.1075/ssol.5.2.01jac

Jacobs, A. M. (2017). Quantifying the beauty of words: a neurocognitive poetics perspective. Front. Hum. Neurosci. 11:622. doi: 10.3389/fnhum.2017.00622

Jacobs, A. M. (2018a). Neuro-Cognitive poetics and computational stylistics. Sci. Study Lit. 8, 164-207. doi: 10.1075/ssol.18002.jac

Jacobs, A. M. (2018b). The gutenberg english poetry corpus: exemplary quantitative narrative analyses. Front. Digit. Humanit. 5:5. doi: 10.3389/fdigh.2018.00005

Jacobs, A. M. (2019). Sentiment analysis for words and fiction characters from the perspective of computational (Neuro-)Poetics. Front. Robot. 6:53. doi: 10.3389/ frobt. 2019.00053

Jacobs, A. M., and Kinder, A. (2019). Computing the affective-aesthetic potential of literary texts. Artif. Intell. 1, 11-27. doi: 10.3390/ai1010002

Jacobs, A. M., Võ. M. L.-H., Briesemeister, B. B., Conrad, M., Hofmann, M. J., Kuchinke, L., et al. (2015). 10 years of BAWLing into affective and aesthetic processes in reading: what are the echoes? Front. Psychol. 6:714. doi: 10.3389/ fpsyg.2015.00714

Jacobs, A. M., Hofmann, M. J., and Kinder, A. (2016a). On elementary affective decisions: to like or not to like, that is the question. Front. Psychol. 7:1836. doi: 10.3389/fpsyg.2016.01836

Jacobs, A. M., Lüdtke, J., Aryani, A., Meyer-Sickendiek, B., and Conrad, M. (2016b). Mood-empathic and aesthetic responses in poetry reception: a model-guided, multilevel, multimethod approach. Sci. Study Lit. 6, 87-130. doi: 10.1075/ssol.6. $1.06 \mathrm{jac}$
Jacobs, A. M., Usée, F., and Lüdtke, J. (2020). Predicting readers' sentiments with Sentiart. (in preparation).

Jakobson, R. (1960). "Closing statement: linguistics and poetics," in Style in language ed. T. A. Sebeok (Cambridge, MA: The MIT Press), 350-377.

Jockers, M. (2017). Introduction to the Syuzhet Package. Available online at: https://cran.r-project.org/web/packages/syuzhet/vignettes/syuzhet-vignette. html (accessed August 29, 2020).

Kuchinke, L., Jacobs, A. M., Grubich, C., Võ, M. L.-H., Conrad, M., and Herrmann, M. (2005). Incidental effects of emotional valence in single word processing: an fMRI study. Neuroimage 28, 1022-1032. doi: 10.1016/j.neuroimage.2005.06 050

Kuiken, D. (2015). The implicit erasure of "literary experience" in empirical studies of literature: comment on "the scientific study of literary experience: sampling the state of the art" by Arthur Jacobs. Sci. Study Lit. 5, 171-177. doi: 10.1075/ ssol.5.2.02kui

Lehne, M., Engel, P., Rohrmeier, M., Menninghaus, W., Jacobs, A. M., and Koelsch, S. (2015). Reading a suspenseful literary text activates brain areas related to social cognition and predictive inference. PLoS One 10:e0124550. doi: 10.1371/ journal.pone.0124550

Lüdtke, J., and Jacobs, A. M. (2015). The emotion potential of simple sentences: additive or interactive effects of nouns and adjectives? Front. Psychol. 6:1137. doi: 10.3389/fpsyg.2015.01137

Lüdtke, J., Meyer-Sickendiek, B., and Jacobs, A. M. (2014). Immersing in the stillness of an early morning: testing the mood empathy hypothesis in poems. Psychol. Aesthet. Creat. Arts 8, 363-377. doi: 10.1037/a0036826

Nicklas, P., and Jacobs, A. M. (2017). Rhetorics, neurocognitive poetics and the aesthetics of adaptation. Poetics Today 38, 393-412.

Willems, R., and Jacobs, A. M. (2016). Caring about Dostoyevsky: the untapped potential of studying literature. Trends Cogn. Sci. 20, 243-245. doi: 10.1016/j. tics.2015.12.009,

Porter, E. (1915). Pollyanna Grows Up. Boston: The Page Company.

Reagan, A. J., Mitchell, L., Kiley, D., Danforth, C. D., and Dodds, P. S. (2016). The emotional arcs of stories are dominated by six basic shapes. EPJ Data Sci. 5:31.

Rowling, J. K. (1997). Harry Potter and the Philosopher's Stone. London: Bloomsbury.

Schroeder, S., Würzner, K. M., Heister, J., Geyken, A., and Kliegl, R. (2015). childLex: a lexical database of German read by children. Behav. Res. Methods 47, 1085-1094. doi: 10.3758/s13428-014-0528-1

Sylvester, T., Braun, M., Schmidtke, D., and Jacobs, A. M. (2016). The Berlin affective word list for children (kidBAWL): exploring processing of affective lexical semantics in the visual and auditory modalities. Front. Psychol. 7:969. doi: 10.3389/fpsyg.2016.00969

Unkelbach, C., von Hippel, W., Forgas, J. P., Robinson, M. D., Shakarchi, R. J., and Hawkins, C. (2010). Good things come easy: subjective exposure frequency and the faster processing of positive information. Soc. Cogn. 28, 538-555. doi: 10.1521/soco.2010.28.4.538

Vonnegut, K. (1981). Palm Sunday. NewYork, NY: Rosetta Books LLC.

Westbury, C., Keith, J., Briesemeister, B. B., Hofmann, M. J., and Jacobs, A. M. (2015). Avoid violence, rioting, and outrage; approach celebration, delight, and strength: using large text corpora to compute valence, arousal, and the basic emotions. Q. J. Exp. Psychol. 68, 1599-1622. doi: 10.1080/17470218.2014. 970204

Xue, S., Lüdtke, J., Sylvester, T., and Jacobs, A. M. (2019). Reading Shakespeare Sonnets?: combining quantitative narrative analysis and predictive modeling an eye tracking study. J. Eye Mov. Res. 12, 1-16. doi: 10.16910/jemr.12.5.2

Xue, S., Jacobs, A. M., and Lüdtke, J. (2020). What is the difference? rereading Shakespeare's Sonnets - an eye tracking study. Front. Psychol. 11:421. doi: 10. 3389/fpsyg.2020.00421

Conflict of Interest: The authors declare that the research was conducted in the absence of any commercial or financial relationships that could be construed as a potential conflict of interest.

Copyright (C) 2020 Jacobs, Herrmann, Lauer, Lüdtke and Schroeder. This is an openaccess article distributed under the terms of the Creative Commons Attribution License (CC BY). The use, distribution or reproduction in other forums is permitted, provided the original author(s) and the copyright owner(s) are credited and that the original publication in this journal is cited, in accordance with accepted academic practice. No use, distribution or reproduction is permitted which does not comply with these terms. 